\title{
Numerical simulation of flow-induced acoustic oscillations around circular cylinders
}

\author{
Serhii Mirnyi ${ }^{1,{ }^{*}}$, Oleg Polevoy ${ }^{1}$, Andrii Zinchenko ${ }^{1}$, Anton Pylypenko ${ }^{2}$, and Vasyl Vlasenko ${ }^{3}$ \\ ${ }^{1}$ Institute of Transport Systems and Technologies of the National Academy of Sciences of Ukraine, \\ 49005, Dnipro, Pisarzhevsky Str., 5, Ukraine \\ ${ }^{2}$ ENGYS North America, MO 63108, Saint Louis, South Sarah Str., 20, USA \\ ${ }^{3}$ Institute of Geotechnical Mechanics named by N. Poljakov of National Academy of Sciences of \\ Ukraine, 49005, Dnipro, Simferopolska Str., 2a, Ukraine
}

\begin{abstract}
Questions of numerical simulation of acoustic oscillations generation modes in the liquid flow around the groups of two and three circular cylinders are considered. In mining industry the processes of hydrodynamic impact on gas-saturated porous media produce significant acoustic emission both at the injection stage and at the liquid discharge stage. Simulation of such kind of acoustic processes is one of the actual problems of theoretical and applied fluid mechanics and under certain assumptions could be reduced to the flow around a group of bodies. Two approaches for numerical simulation of the acoustic oscillations generation induced by the flow around circular cylinders based on numerical solution of the Navier-Stokes equations for compressible and incompressible flows closed by differential model of turbulence and complemented by acoustic analogy equations have been developed. For laminar flows, eight different modes that fundamentally differ both in the flow structure and in the frequency spectrum of parameter oscillations have been identified. For turbulent flows, the classification criteria for the three main frequency modes are presented. Acoustic data are obtained using the Direct Noise Computation technology and acoustic analogies as well.
\end{abstract}

\section{Introduction}

Many technical problems require deep study of acoustic processes induced by flows around a group of objects, like flows around power lines, urban high-rise buildings [1,2] on macro level and bubble- or particle-saturated flows in small cavities on micro level. In mining industry there is lot of methods for processing the outburst hazardous coalbeds with the liquids [3], such as hydrodynamic impact on gas-saturated porous media [4,5]. The exposure process is accompanied by active acoustic emission both at the injection stage and at the liquid discharge stage. Simulation of acoustic emission in such kind of processes is one of the actual problems of theoretical and applied fluid mechanics and under certain assumptions could be reduced to flow around a group of bodies.

The flow regimes around the standalone cylinder have been well studied for now. The

*Corresponding author: mirnyss@gmail.com 
laminar, subcritical and supercritical flow regimes are comprehensively described and empirical dependences for the fundamental oscillation frequencies are obtained [6-9].

At the same time the flow regimes around groups of bodies are much more complicated. The theoretical interest in this class of flows is stipulated by many factors such as complex spatial geometry created by a group of bodies; viscous-inviscid interaction accompanied by the flow separation; unsteady turbulence; nonlinear dependencies of the determining and resulting parameters. The resulting pressure pulsations could be both periodic and stochastic. Generated acoustic fields, the overall sound pressure level is difficult to estimate even a priori $[7,10]$.

The practical interest in this problem is based on the fact that by changing the size of cylinders, the distance between them, the location angle of the group relative to the incoming flow, it is possible to achieve the desired intensity and direction of acoustic oscillations generated or damping of the oscillation amplitude conversely. The study of the regimes of acoustic vibrations generation during the air flow around the group of bodies will contribute to the improvement of methods of hydrodynamic impact on gas-saturated porous media and development of environmentally friendly and effective novel methods for opening outburst hazardous coalbeds and coal mining.

Experimental studies of aerodynamics and acoustics of closely spaced bodies are difficult and expensive, especially if the obstacles in the flow have large sizes. The necessity of such research at the preliminary stage of industrial facilities design makes the problem even more complicated. Using traditional analytical methods of classical acoustics is also limited due to the complex geometry and non-periodic spatial distribution of fluid parameters.

Therefore, the most promising direction in the study of acoustic processes in a flow around a group of bodies is the computational experiments based on the numerical solution of the Navier-Stokes equations closed by differential models of turbulence and equations of acoustic analogy. Numerical simulation requires an adequate mathematical formulation of the problem, the choice of efficient discretization algorithms, visualization methods and the post processing for the data obtained.

The main goal of the paper is the investigation of the acoustic oscillation's generation regimes in the air flow around groups of bodies consisting of two and three circular cylinders. The considered flows diagrams are shown on Fig. 1.
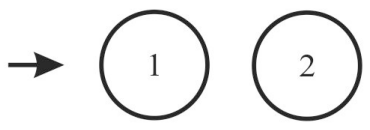

a)
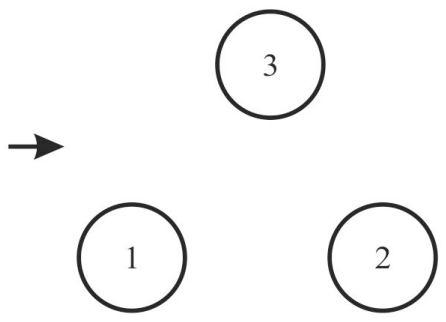

c)

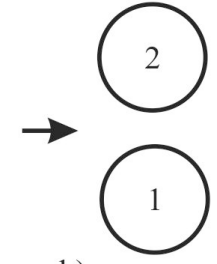

b)

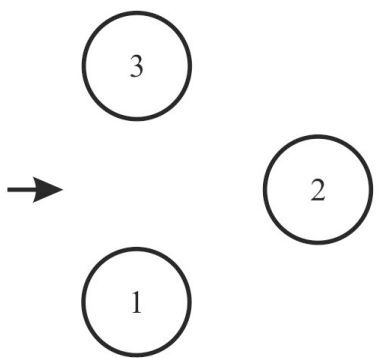

d)

Fig. 1. Schematic representation of two $(a, b)$ and three $(c, d)$ circular cylinders in the air flow. 


\section{Methods}

Two approaches for numerical simulation of acoustic oscillations in air flow have been considered.

The first of them is based on unsteady Reynolds-averaged Navier-Stokes equations (URANS) of compressible flows, written in an arbitrary coordinate system $(\xi, \eta)$. The governing equations system in non-dimensional form can be written as $[11,12]$ :

$$
\frac{\partial \mathbf{q}}{\partial t}+\frac{\partial \mathbf{E}}{\partial \xi}+\frac{\partial \mathbf{F}}{\partial \eta}=\frac{1}{\operatorname{Re}}\left(\frac{\partial \mathbf{E}_{v}}{\partial \xi}+\frac{\partial \mathbf{F}_{v}}{\partial \eta}\right)
$$

where $\mathbf{q}=(\rho, \rho u, \rho v, e)^{\mathrm{T}}-$ vector of conservative variables, $\mathbf{E}, \mathbf{F}, \mathbf{E}_{v}, \mathbf{F}_{v}-$ vectors of convective and dissipative fluxes; $t$ - time; $u, v$ - velocity vector components in $x$ and $y$ directions; $\rho, e$-density and total energy per unit air volume; Re - Reynolds number. For system of equations (1) the boundary conditions of adhesion and adiabaticity are used on solid body surface. The parameters at the outer boundary are calculated using Riemann invariants $[11,12]$ as a result of free stream flow interaction with disturbances from the computational domain.

The system of equations (1) is closed by the Spalart-Allmaras differential turbulence model [13]. The implicit numerical algorithm is based on the Roe scheme [14] and has a second order of accuracy in time and space. Details of construction of the numerical algorithm and its verification on subsonic and transonic flow around airfoils, including the regime of shock waves self-induced oscillations, are presented in [12].

Acoustic data could be obtained using Direct Noise Computation (DNC) technology. For unsteady flows the averaged stationary pressure field $\bar{p}$ was calculated over a certain time interval $T$ equal to $20-25$ periods of instantaneous pressure value $p(t)$ oscillations. Then the sound pressure $p_{\text {RMS }}$ field was determined as the RMS (Root Mean Square) of the instantaneous sound pressure $p_{a}(t)=p(t)-\bar{p}$ over a specified period of time $T$ :

$$
p_{\mathrm{RMS}}=\sqrt{\frac{1}{T} \int_{0}^{T} p_{a}^{2}(t) d t}=\sqrt{\frac{1}{T} \int_{0}^{T}(p(t)-\bar{p})^{2} d t} .
$$

The intensity of acoustic noise is determined by the logarithmic scale of sound pressure (SPL - Sound Pressure Level) in dB:

$$
L_{\mathrm{SPL}}=10 \cdot \lg \left(p_{\mathrm{RMS}}^{2} / p_{0}^{2}\right)=20 \cdot \lg \left(p_{\mathrm{RMS}} / p_{0}\right)
$$

where $p_{0}=2 \cdot 10^{-5} \mathrm{~Pa}$ - sound pressure reference. Relations (2), (3) correspond to the international standard ISO 3740:2000 [15].

A test calculation setup of the acoustic oscillations generation was taken from experimental study [16]. The flow velocity was of $68 \mathrm{~m} / \mathrm{s}$, which corresponds to the Mach number $\mathrm{M}_{\infty}=0.2$ of the incoming flow. For cylinder diameter of $D=38 \mathrm{~mm}$ the corresponding Reynolds number was $\mathrm{Re}=1.8 \cdot 10^{5}$, i.e. the flow was in subcritical mode.

The turbulent viscosity contours presented in Fig. 2 well reflect the vortex structure in the wake of the cylinder. Spectral analysis of the frequency distribution of the calculated intensity of acoustic noise for a control point located at the distance $L=35 D$ from the center of the cylinder at an angle of $90^{\circ}$ to the incoming flow is shown in Fig. 3 in comparison with experimental data [16]. The OASPL (Overall Sound Pressure Level) at this point is $118.8 \mathrm{~dB}$ and represented good agreement with experimental value of $117 \mathrm{~dB}$.

Direct computation of acoustic noise based on the numerical solution of unsteady viscous compressible flow equations is a natural approach to the solution of acoustics 
problems. However, as the practice of calculations shows, at low flow velocities corresponded to small Mach numbers $\left(\mathrm{M}_{\infty}<0.1\right)$, the numerical solution of equations (1) may contain nonphysical oscillations caused by a large difference between the flow velocity and the sound speed [17].

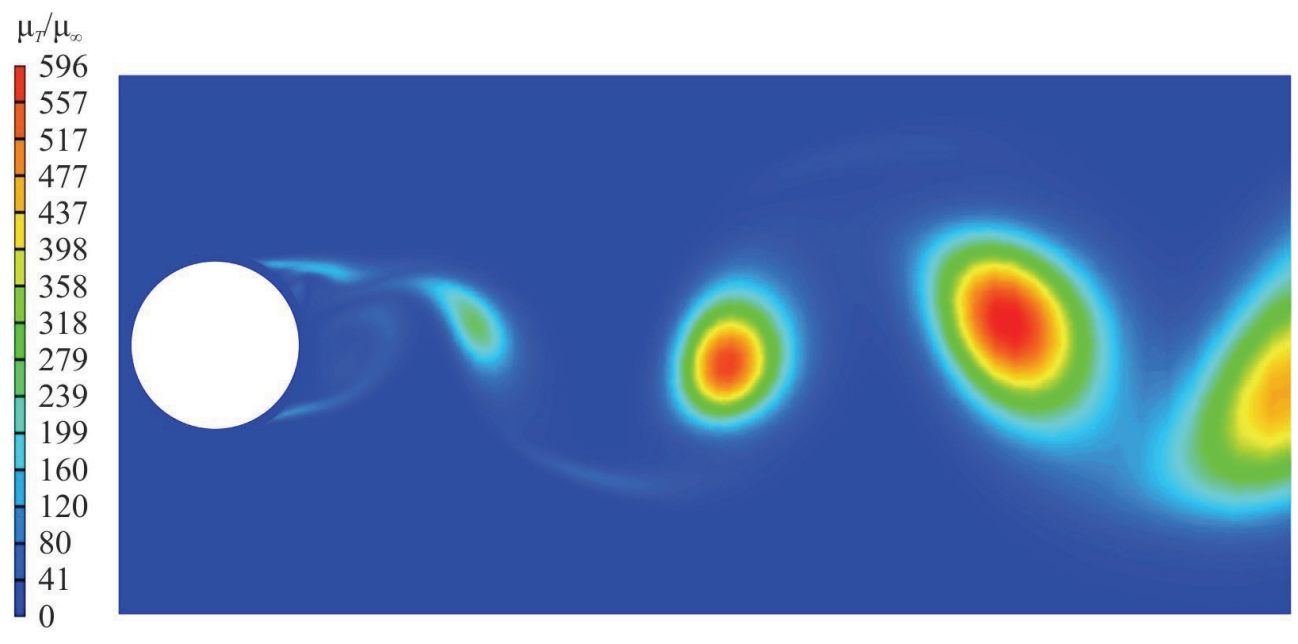

Fig. 2. Distribution of non-dimensional turbulent viscosity $\mu_{T} / \mu_{\infty}$ in the flow around standalone circular cylinder, where $\mu_{\infty}$ is the molecular viscosity of free stream.

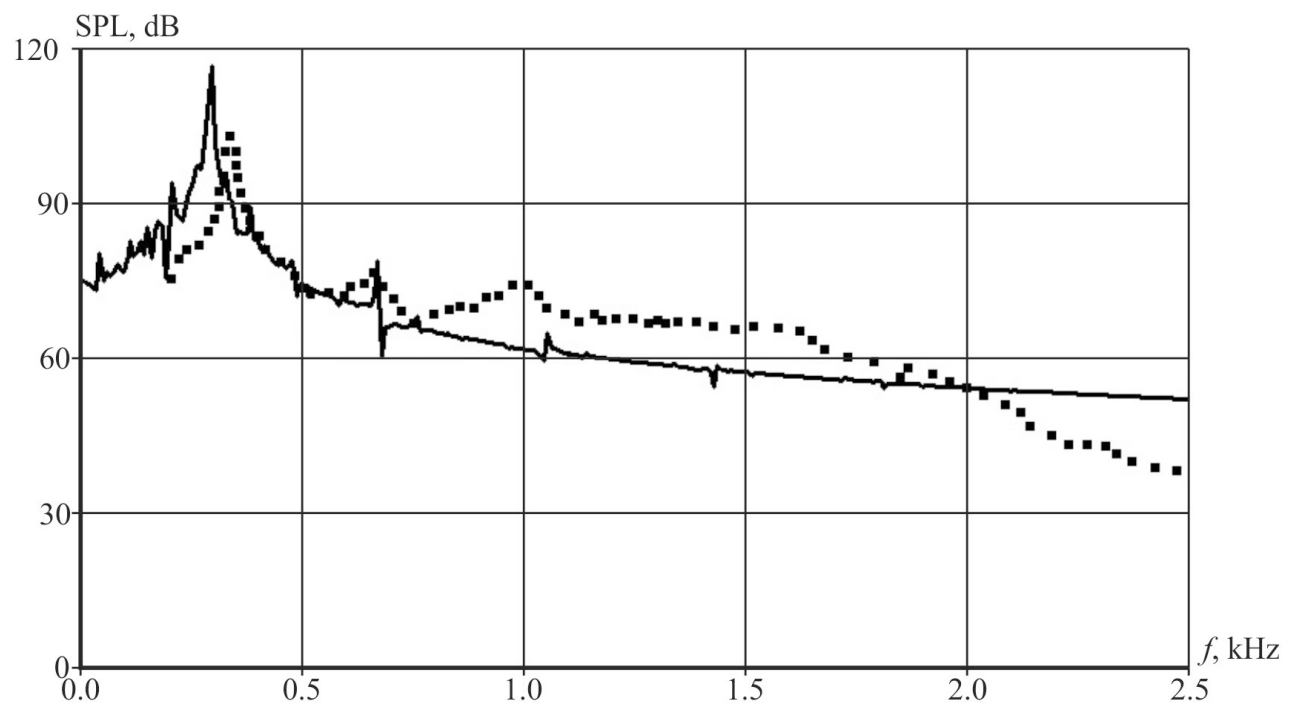

Fig. 3. The SPL frequency distribution in control point at flow around a standalone circular cylinder. - - experiment [16]; - this work.

An alternative approach for the low velocity range is the numerical solution of unsteady Reynolds-averaged Navier-Stokes equations (URANS) for incompressible fluid in nondimensional form:

$$
\frac{\partial u}{\partial x}+\frac{\partial v}{\partial y}=0
$$




$$
\begin{aligned}
& \frac{\partial u}{\partial t}+\frac{\partial\left(u^{2}\right)}{\partial x}+\frac{\partial(u v)}{\partial y}=-\frac{1}{\rho} \frac{\partial p}{\partial x}+\frac{1+\mu_{T} / \mu_{\infty}}{\rho \operatorname{Re}}\left(\frac{\partial^{2} u}{\partial x^{2}}+\frac{\partial^{2} u}{\partial y^{2}}\right) \\
& \frac{\partial v}{\partial t}+\frac{\partial(u v)}{\partial x}+\frac{\partial\left(v^{2}\right)}{\partial y}=-\frac{1}{\rho} \frac{\partial p}{\partial y}+\frac{1+\mu_{T} / \mu_{\infty}}{\rho \operatorname{Re}}\left(\frac{\partial^{2} v}{\partial x^{2}}+\frac{\partial^{2} v}{\partial y^{2}}\right)
\end{aligned}
$$

The boundary conditions similar in physical sense to compressible flows are used for the system (4) - (6).

URANS equations could be closed either with Reynolds stress model, either using the Boussinesq hypothesis. In present paper the $k-\omega$ SST (Menter's Shear Stress Transport) turbulence model is used to simulate the flow around a group of circular cylinders [18].

At the same time, direct use of equations (4), (5) combined with DNC technology is not methodologically reasonable, since equations (4), (5) do not take into account fluctuations of air density.

Although, there are lots of methods developed to simulate the acoustic noise generation in incompressible fluid. The most common ones are the acoustic analogies by Curle [19] and Ffowcs Williams-Hawkings [20] which are based on the acoustic analogy by Lighthill $[21,22]$.

In present paper the Curle acoustic analogy [19] is used. Acoustic pressure could be calculated as follows:

$$
p_{a}=\frac{4 \pi}{c_{0}} \frac{\overrightarrow{\mathbf{d}}}{|\overrightarrow{\mathbf{d}}|^{2}} \frac{d F}{d t},
$$

where $p_{a}$ - acoustic pressure calculated by Curle analogy, $\mathrm{Pa} ; c_{0}-$ sound speed, $\mathrm{m} / \mathrm{s} ; \overrightarrow{\mathbf{d}}-$ distance vector to the observer, $\mathrm{m} ; F$ - aerodynamic force, N.

During the computational experiments, the governing equations systems were discretized based on the finite volume approach and solved using the OpenFOAM opensource software [23]. The verification of methodology presented was based on simulating flows around two- and three-dimensional bodies [24].

For standalone cylinder, the test calculation is performed for the supercritical Reynolds number $1.810^{6}$. The flow velocity was corresponded to a Mach number of 0.128 . The instantaneous distribution of acoustic pressure, calculated using the Curle analogy is shown in Fig. 4. The acoustic pressure pattern near the cylinder indicates that the sound induced by variable pressure on the cylinder surface, corresponds to the sound generation by a dipole, which agrees well with known data $[19,20]$.

Acoustic pressure, $\mathrm{Pa}$
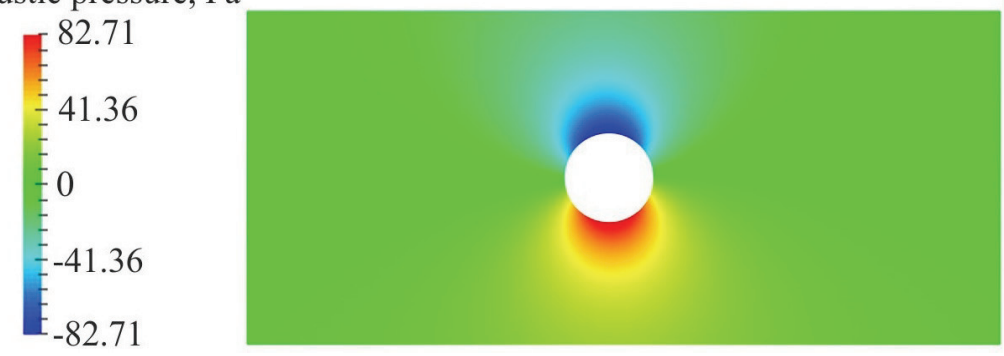

Fig. 4. Distribution of instantaneous acoustic pressure, calculated using the Curle analogy in the flow around a standalone circular cylinder. 


\section{Results and discussion}

Computational experiments on the generation of acoustic oscillations were carried out for both a laminar flow with a Reynolds number, calculated by cylinder diameter $D$, $\mathrm{Re}_{D}=120$ and turbulent flow with a number $\mathrm{Re}_{D}=1.66 \cdot 10^{5}$.

For a tandem of cylinders, the first cylinder was located at the origin of coordinates. The position of the second cylinder was defined by the gap between the cylinders $h$, and the angle $\theta$ between the positive direction of the axis $O x$ and the straight line connecting the centers of the cylinders. The numerical simulation was performed as of $\theta=0^{\circ}-90^{\circ}$ and $h=(0.2-5.0) D$ (Fig. 5 a, b).

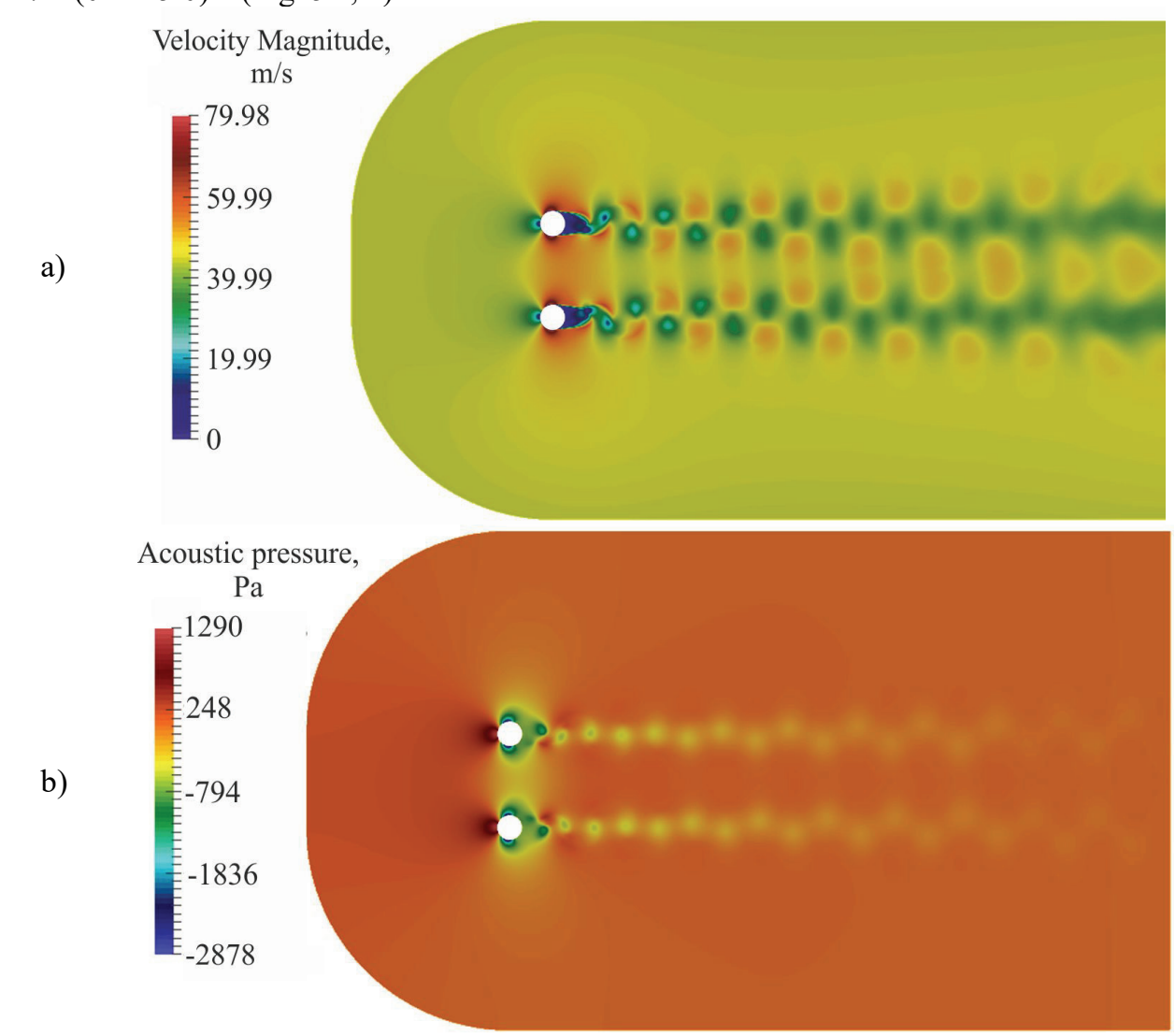

Fig. 5. Distributions of the velocity vector modulus (a) and the instantaneous acoustic pressure (b) in the flow around a tandem of circular cylinders with a Reynolds number $\operatorname{Re}_{D}=1.66 \cdot 10^{5}$.

In all cases considered the flow regime was transient, and flow parameters were significantly depended on the relative position of the cylinders. Fig. 5 presents the instantaneous results for the turbulent flow around the tandem of cylinders at $h=3.0 D$ and $\theta=90^{\circ}$.

Since Curle acoustic analogy is based on the time variation of the aerodynamic force $d F / d t$, special attention was paid to the changes in the integral aerodynamic characteristics - the drag coefficient $C_{x}$, the lift coefficient $C_{y}$ for each cylinder separately $\left(C_{x 1}, C_{y 1}, C_{x 2}, C_{y 2}\right)$, and in the case when cylinders are considered as one fixed system as well $\left(C_{x}, C_{y}\right)$. The properties of the time dependences of the aerodynamic coefficients were used as the basis for the classification criteria of the regimes of acoustic oscillations generation. 
For each computational case, a non-stationary lift-drag polar was constructed, which is a curve, the coordinates of each point of which correspond to the values $\left(C_{x}, C_{y}\right)$ at a certain time moment. By the shape of non-stationary polar, it is possible to determine whether the process is periodic or not. Non-stationary lift-drag polar has the shape of closed curve when the flow around the cylinder is a non-stationary periodic process and the vortices are detached from the cylinders with a constant frequency. In that case the changes of aerodynamic coefficients would be periodic also. If the flow around the cylinders is an aperiodic process, the non-stationary polars will be open curves.

Using the fast Fourier transformation the number of frequencies, the value of the Strouhal numbers, the amplitudes of the aerodynamic coefficients oscillations were determined. The average values of drag and lift coefficients were also determined. Using the values of maximum amplitudes, the phases of vortices formation and flow separation could be obtained. If the formation of vortices on the surface of cylinders occurs simultaneously, then the total maximum amplitude of lift coefficient oscillations will be close to the sum of the maximum amplitudes of the oscillations of individual cylinders lift coefficients. If the vortices formation occurs in antiphase, then the total maximum amplitude of oscillations of the lift coefficient will be significantly less than the maximum amplitudes of the cylinders lifting force coefficients oscillations.

The presence of a second cylinder in the fluid flow results to significant changes in frequencies. When the angle $\theta$ and the gap $h$ between the cylinders are changed, the oscillations of the aerodynamic coefficients may have several distinct frequencies. This fact demonstrates the significant difference from the flow around a standalone cylinder.

The analysis of the obtained results allowed us to identify eight different regimes of the flow around two cylinders taking into account their different relative positions. The feature classification of these regimes are summarized in Table. 1.

Table 1. Flow regimes around two cylinders.

\begin{tabular}{|c|l|}
\hline Regime & \multicolumn{1}{c|}{ Description } \\
\hline Regime 1 & $\begin{array}{l}\text { Stationary regime for the first cylinder, the drag coefficient of the second } \\
\text { cylinder is negative. }\end{array}$ \\
\hline Regime 2 & $\begin{array}{l}\text { Stationary regime for the first cylinder, the drag coefficient of the second } \\
\text { cylinder is positive. }\end{array}$ \\
\hline Regime 3 & $\begin{array}{l}\text { Non-stationary regime, polar curves are closed, there is a small number of } \\
\text { frequencies (1-3), the oscillation phases are the same or close. }\end{array}$ \\
\hline Regime 4 & $\begin{array}{l}\text { Non-stationary regime, polar curves are closed, there is a small number of } \\
\text { frequencies (1-3), oscillations occur in antiphase. }\end{array}$ \\
\hline Regime 5 & $\begin{array}{l}\text { Non-stationary regime, polar curves are closed, the average number of } \\
\text { frequencies (3-6) and the oscillation phases are the same or close. }\end{array}$ \\
\hline Regime 6 & $\begin{array}{l}\text { Non-stationary regime, polar curves are closed, there is a large number of } \\
\text { frequencies, the oscillation phases are the same or close. }\end{array}$ \\
\hline Regime 7 & $\begin{array}{l}\text { General non-stationary regime, polar curves are closed, there is a large } \\
\text { number of frequencies, oscillations occur in antiphase. }\end{array}$ \\
\hline Regime 8 & $\begin{array}{l}\text { Chaotic non-stationary mode, unclosed polar curves, there is a large } \\
\text { number of frequencies, oscillations are aperiodic. }\end{array}$ \\
\hline
\end{tabular}

During the computational experiments for the flow around the group of three cylinders, the cylinders were located at the vertices of an equilateral triangle (Fig. $1 \mathrm{c}, \mathrm{d}$ ). The simulation was carried out for the gaps between the cylinders equal to $(0.5-3.0) D$ and angles in range of $\theta=0^{\circ}-90^{\circ}$.

The flow around the cylinders has a complex structure. Flow separation occurs from all three cylinders and therefore all bodies have an influence to the formation of the vortex 
street behind the group. Fig. 6 shows the distributions of the velocity vector magnitude, the instantaneous acoustic pressure and the kinetic energy of turbulence for the angle $\theta=0^{\circ}$ and $h=3.0 D$.

During the numerical experiments, the significant influence of the position of cylinders in the group on their aerodynamic coefficients and amplitude-frequency characteristics was observed. The maximum amplitudes of the oscillations of the coefficients and the corresponding frequencies significantly differ from the amplitudes and oscillations of the aerodynamic coefficients for standalone cylinder. In series of cases, the noticeable increase in the number of frequencies of aerodynamic coefficients oscillation is observed. In general case, these dependencies correspond to Lissajous figures of complex shape (Fig. 7).

a)

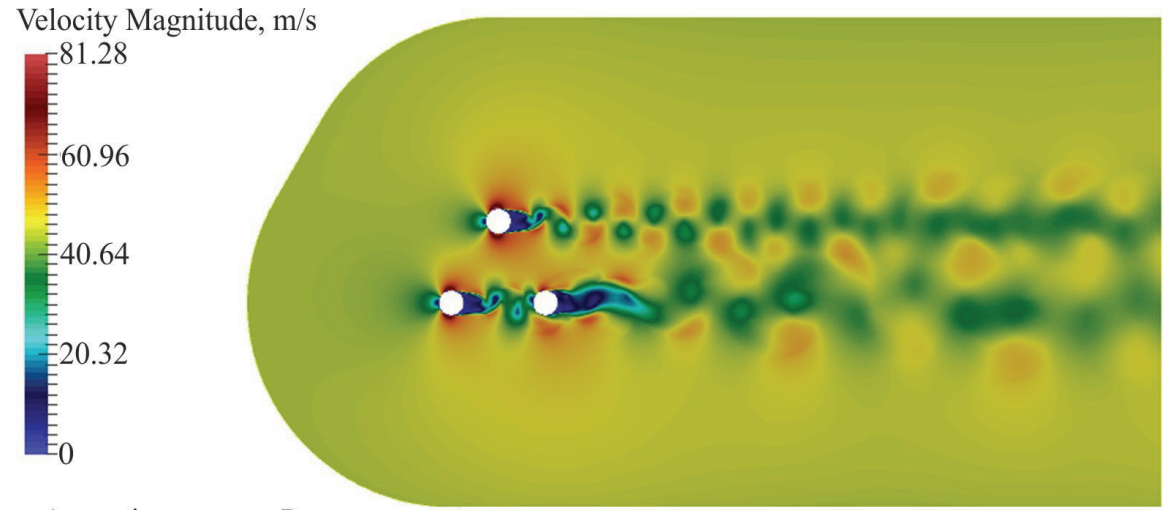

b)

Acoustic pressure, $\mathrm{Pa}$

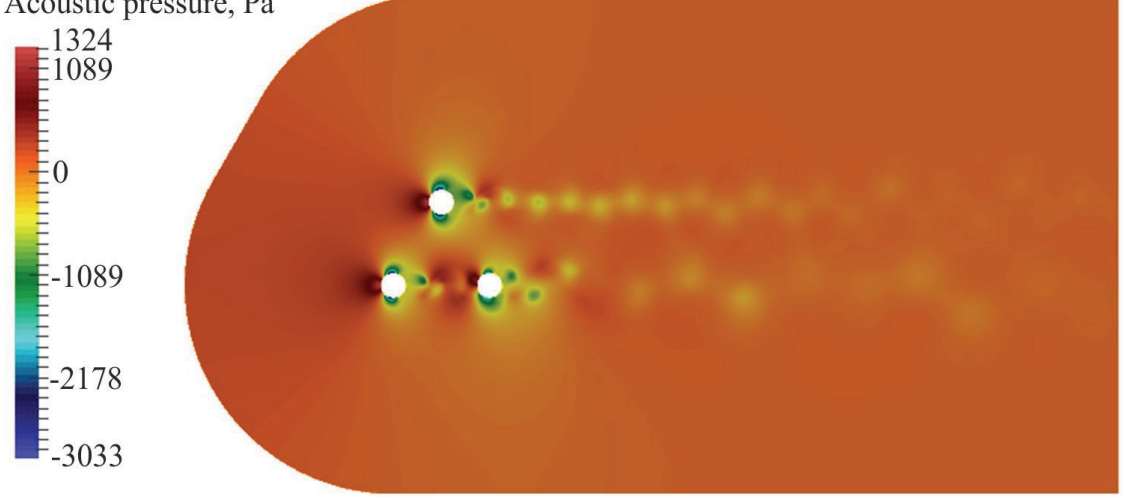

c)

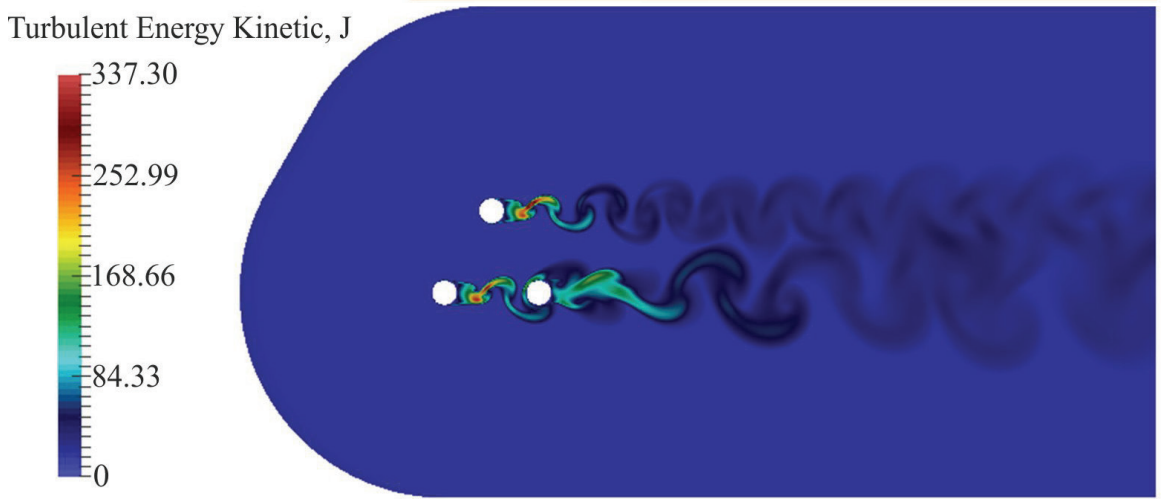

Fig. 6. Distributions of the velocity vector modulus (a), the instantaneous acoustic pressure (b) and kinetic energy of turbulence (c) in the flow around three circular cylinders with Reynolds number $\operatorname{Re}_{D}=1.66 \cdot 10^{5}$. 


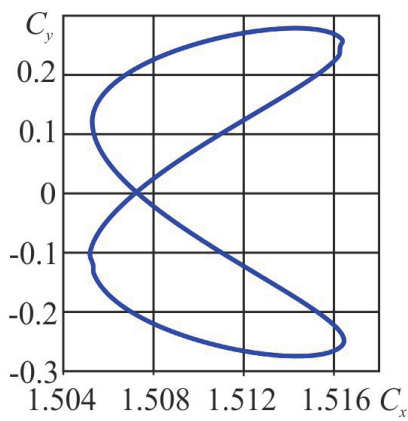

a)

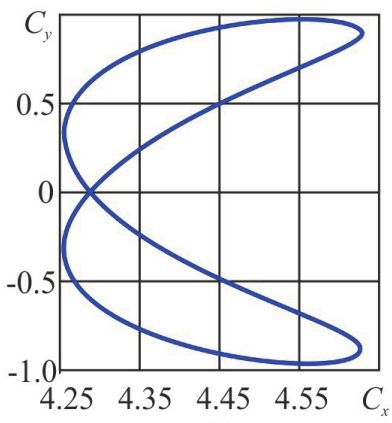

b)

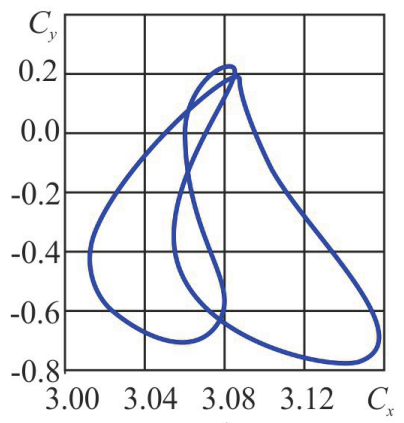

c)

Fig. 7. Transient dependencies of lift coefficient $C_{y}$ from drag coefficient $C_{x}$ for single cylinder (a), cylinders in staggered arrangement $h=0.2 D, \theta=90^{\circ}$ (b), cylinders in staggered arrangement $h=0.8 D, \theta=45^{\circ}(\mathrm{c})$.

Fig. 7 b shows that dependency of $C_{y}\left(C_{x}\right)$ for tandem arrangement with small distances between two cylinders is similar to the one for single cylinder (Fig. 7 a). Increasing the gap between the cylinders and angle of the incoming flow stipulates this dependency become different and more complicated (Fig. 7 c). These changes correspond to changes in flow picture and flow regimes.

Three main flow regimes for the group of three cylinders can be distinguished: the low-frequency (1-3 harmonics present); the multi-frequency, ordered (there are more than 3 harmonics); and multi-frequency disordered (there are more than 3 frequencies).

\section{Conclusions}

Two approaches for numerical simulation of the acoustic oscillations generation in air flow around circular cylinders have been developed based on numerical solution of the NavierStokes equations for compressible and incompressible flows closed by differential model of turbulent viscosity and complemented by acoustic analogy equations. Developed methods have been verified on experimental data of other authors.

Computational experiments were carried out to determine acoustic oscillations in the flow around groups of bodies consisting of two and three circular cylinders. Classification criteria to identify various flow regimes and the generation of acoustic noise have been formulated.

Significant mutual influence of the cylinders in the group on their aerodynamic and acoustic characteristics was observed. The maximum oscillations amplitudes of the aerodynamic coefficients and the corresponding frequencies significantly differ from the corresponding parameters of standalone cylinder. Generally these dependencies have been represented by complex shaped Lissajous figures.

The considered model could be used to describe the process of coal particles movement in a water flow during hydrodynamic impact on gas-saturated porous media. The study of acoustic oscillations induced by particle motion allows improving the quality analysis of acoustic emission in mining processes. This makes it possible to obtain the new details of fissure formation in coalbeds as a result of hydrodynamic impact on it, and develop new methods of vibroacoustic diagnostics based on acoustic emission in predetermined direction. 


\section{References}

1. R. Ohayon, C. Soize, Structural Acoustics and Vibration: Mechanical Models, Variational Formulations and Discretization (Academic Press, 1997)

2. I.L. Vér, L.L. Beranek, Noise and Vibration Control Engineering: Principles and Applications (Wiley; 2 edition, 2005)

3. SOU 10.1.00174088.001. (2004). Dehazyfikatsiia vuhilnykh shakht. Vymohu do sposobiv ta skhem dehazatsii. Kyiv: Minpalyvenerho Ukrainy

4. Sofiyskiy, K.K., Gavrilov, V.I., Zhitlenok, D.M., Vlasenko, V.V., Petukh, A.P. (2015). Gidrodinamicheskiye sposoby vozdeystviya na napryazhennyye gazonasyshchennyye ugolnyye plasty. Donetsk: Skhidnyi vydavnychyi dim

5. V.Vlasenko, K. Dudlia, M. Kyrychenko. Mathematical model of the cracking process in the coal-rock massif under hydrodynamic impact. E3S Web of Conferences, International Conference Essays of Mining Science and Practice, 109 (2019). https://doi.org/10.1051/e3sconf/201910900111

6. H. Schlichting, K. Gersten, Boundary-Layer Theory (Springer-Verlag Berlin Heidelberg, 2017)

7. B.M. Sumer, Hydrodynamics around cylindrical structures (Denmark, Technical University of Denmark, 2006)

8. U. Fey, M. König, H. Eckelmann, A new Strouhal-Reynolds-number relationship for the circular cylinder in the range $47<R e<23105$, Physics Fluids, 10 (1998)

9. A.I. Voloshin, V.N. Poturaev, B.V. Ponomarev. (1989). One-dimensional flow of a twophase medium. Soviet Applied Mechanics, 25 (8)

10. B.S. Carmo, On wake interference in the flow around two circular cylinders: direct stability analysis and flow-induced vibrations ( $\mathrm{PhD}$ thesis, Department of Aeronautics, Imperial College, London, 2009)

11. T.H. Pulliam, Efficient solution methods for the Navier-Stokes equations. Lecture notes for the von Karman Institute for Fluid Dynamics (Belgium, Lecture Series Von Karman Institute, 1985)

12. A.O. Pylypenko, O.B. Polevoy, O.A. Prykhodko, Numerical simulation of Mach number and angle of attack influence on regimes of transonic turbulent flows over airfoils, TsAGI Science Journal, 43(1) (2012)

13. P.R. Spalart, S.R. Allmaras, A one-equation turbulence model for aerodynamic flow (AIAA Paper 92-439, 1992)

14. P.L. Roe, Approximate Riemann solvers, J. Comp/ Phys, 43 (1981)

15. ISO 3740:2000-11 (E) Acoustics - Determination of sound power levels of noise sources - Guidelines for the use basic standards.

16. J.D. Revell, R.A. Prydz, A.P. Hays, Experimental study of aerodynamic noise vs drag relationships for circular cylinders (AIAA Paper 77-1292, 1977)

17. J.C. Tannehill, D.A. Anderson, R.H. Pletcher, Computational fluid mechanics and heat transfer (Taylor \& Francis, New York, Second edition, 1997)

18. F.R. Menter, Improved two-equation $k-\omega$ turbulence models for aerodynamic flows (NASA-92-tm-103975, 1992)

19. N. Curle, The Influence of Solid Boundaries upon Aerodynamic Sound, Proceedings of the Royal Society A: Mathematical, Physical and Engineering Sciences, 231 (1187) (1955)

20. J.E. Ffowcs Williams, D.L.Hawkings, Sound Generated by Turbulence and Surfaces in Unsteady Motion, Philosophical Transactions of the Royal Society, A264 (1151) (1969)

21. M.J. Lighthill, On sound generated aerodynamically I. General theory. Proc. of the Royal Society of London, A211 (1952) 
22. M.J. Lighthill, On sound generated aerodynamically II. General theory. Proc. of the Royal Society of London, A222 (1954)

23. H. G. Weller, G. Tabor, H. Jasak, C. Fureby, A tensorial approach to computational continuum mechanics using object-oriented techniques, Computers in Physics, Vol. 12, No.6 (1998)

24. Mirny, S., Redchyts, D. (2019) Numerical simulation of the viscous incompressible flow around of the group of two bodies. Systemnye tekhnologii [System technologies], 3(122), 117-132 